\title{
Interface Phenomena and Interaction Energy at the Surface of Electroconductive Solids
}

\author{
Taras Nahirnyj ${ }^{1,2}$ and Kostiantyn Tchervinka ${ }^{3}$ \\ ${ }^{1}$ Centre of Mathematical Modelling, National Academy of Science of Ukraine \\ ${ }^{2}$ University of Zielona Góra \\ e-mail: tnagirny@yahoo.com \\ ${ }^{3}$ Ivan Franko National University of Lviv, Ukraine \\ e-mail:K.Tchervinka@gmail.com
}

(Received: 14 May 2008; published online: 20 November 2008)

\begin{abstract}
For the local gradient approach in thermomechanics, the regularities of nearsurface nonhomogeneity in nonferromagnetic electroconductive solids, including the size effect of the ultimate stress limit are studied. It is noted that surface values of interaction energy and thermodynamic electric potential are determined by physical and geometrical properties of a body.
\end{abstract}

Key words: local gradient approach in thermomechanics, interface phenomena, interaction energy, size effects, strength

\section{INTRODUCTION}

One of the major tasks of the solid mechanics is predicting the deformation, strength and other parameters of real constructions and devices that are exposed to the complex action of the environment. Such prognosis is based on the use of proper mathematical models that should take into account properties and structure of the material as well as nature of external effects. Proper consideration of the effects of local nonhomogeneity is especially topical in connection with a wide practical application of thin films and fibres, fine laminations, and the like. It is known that such elements feature proportionality of surface and volume factor components in the internal energy. Nearsurface heterogeneity substantially influences crack propagation and brittle fracture, flow deformation and, so on. In the specialistic literature there are some approaches to describe nearsurface heterogeneity within nonferromagnetic electroconductive solids and solid solution models that are based on the concept of an electron body structure with a double electric layer [1, 2]. For such model approach the nearsurface heterogeneity of different physical fields is considered as a result of electric potential heterogeneity.

Local gradient models allow describing the effects of local heterogeneities and the influence of different physical nature fields on them. Thus, such models are appropriate to describe the above phenomena. In works [3-6] and others, the basic relations of the local gradient approach in thermodynamics are presented, and the regularities of nearsurface nonhomogeneity in thermoelastic solids and solid solutions are investigated. The approach has been set up on the basic principles of thermodynamics of irreversible processes. It allows to account for the influence of different physical nature fields on mechanical behaviour of the body.

In this paper the basic relations of the nonferromagnetic electroconductive solid are presented for the local gradient approach, and the regularities of nearsurface heterogeneity and the size effect of the ultimate stress limit are investigated.

\section{LOCAL GRADIENT APPROACH IN THERMOMECHANICS OF ELECTROCONDUCTIVE SOLIDS}

The physical and mechanical fields should follow the laws of thermodynamics and balance equations. The starting point for the solid mechanics model construction is usually the equation of total energy balance. We suppose that the total energy $E$ can be expressed as a sum of internal $U$, kinetic $K$, and electromagnetic field $U_{e}$ energies

$$
E=U+K+U_{e},
$$


where the latter depends on intensities of electric $\vec{E}$ and magnetic $\vec{H}$ fields.

$$
U_{e}=\left(\varepsilon_{0} \vec{E}^{2}+\mu_{0} \vec{H}^{2}\right) / 2
$$

satisfies the following balance equation

$$
\frac{\partial U_{e}}{\partial \tau}=\frac{1}{\mu_{0}} \vec{\nabla} \cdot(\vec{E} \times \vec{B})+\left(\vec{J}_{\omega}+\omega \vec{v}\right) \cdot \vec{E},
$$

where $\tau$ is time, $\vec{B}=\mu_{0} \vec{H}, \vec{J}_{\omega}$ is the vector of electric charge flux, $\omega$ is the electric charge density, and $\vec{v}$ is the barycentric velocity.

The balance equation for the total energy in the local form for the geometric linear approach is as follows

$$
\frac{\partial E}{\partial \tau}=\vec{\nabla} \cdot\left(\hat{\sigma} \cdot \vec{v}-T \vec{J}_{s}-H \vec{J}_{m}-\Phi \vec{J}_{\omega}+\frac{1}{\mu_{0}} \vec{E} \times \vec{B}\right) .
$$

Here $\hat{\sigma}$ is the Cauchy stress tensor, $T$ is temperature, $H$ is the chemical, and $\Phi$ is the thermodynamical electric potentials [1], $\vec{J}_{s}$ and $\vec{J}_{m}$ are the vectors of the entropy and mass fluxes.

The balances of momentum $\vec{k}_{v}$, entropy $S$, mass density $\rho$, and electric charge density $\omega$ are

$$
\begin{gathered}
\frac{\partial \vec{k}_{v}}{\partial \tau}=\vec{\nabla} \cdot \hat{\sigma}+\vec{F}_{e}, \quad \frac{\partial S}{\partial \tau}=-\vec{\nabla} \cdot \vec{J}_{s}+\sigma_{s}, \\
\frac{\partial \rho}{\partial \tau}=-\vec{\nabla} \cdot \vec{J}_{m}, \quad \frac{\partial \omega}{\partial \tau}=-\vec{\nabla} \cdot \vec{J}_{\omega},
\end{gathered}
$$

where $\vec{F}_{e}=\left(\vec{J}_{\omega}+\omega \vec{v}\right) \times \vec{B}+\omega \vec{E}$ is ponderomotive force and $\sigma_{s}$ is entropy production.

Maxwell's equations can be written in the local form as $[1,2]$

$$
\begin{gathered}
\vec{\nabla} \times \vec{E}=-\frac{\partial \vec{B}}{\partial \tau}, \quad \vec{\nabla} \times \vec{B}=\mu_{0} \varepsilon_{0} \frac{\partial \vec{E}}{\partial \tau}+\mu_{0}\left(\vec{J}_{\omega}+\omega \vec{v}\right), \\
\vec{\nabla} \cdot \vec{B}=0, \quad \varepsilon_{0} \vec{\nabla} \cdot \vec{E}=\omega .
\end{gathered}
$$

The local gradient approach in thermomechanics is based on presenting the flux $\vec{J}_{k}$ as a sum of the irreversible $\vec{j}_{k}$ and the reversible $\partial \vec{\pi}_{k} / \partial \tau$ components $(k=\{s, m, \omega\})$. In this particular model we assume

$$
\vec{J}_{s}=\vec{j}_{s}, \quad \vec{J}_{\omega}=-\frac{\partial \vec{\pi}_{\omega}}{\partial \tau}+\vec{j}_{\omega}, \quad \vec{J}_{m}=-\frac{\partial \vec{\pi}_{m}}{\partial \tau}
$$

that will modify the equations of mass and charge balance to the form

$$
\frac{\partial}{\partial \tau}\left(\rho-\vec{\nabla} \cdot \vec{\pi}_{m}\right)=0, \quad \frac{\partial}{\partial \tau}\left(\omega-\vec{\nabla} \cdot \vec{\pi}_{\omega}\right)=-\vec{\nabla} \cdot \vec{j}_{\omega} .
$$

From the above relations we obtain the equation of internal energy balance

$$
\begin{aligned}
\frac{\partial U}{\partial \tau} & =T \frac{\partial S}{\partial \tau}+H \frac{\partial \rho}{\partial \tau}+\Phi \frac{\partial \omega}{\partial \tau}+\hat{\sigma}: \frac{\partial \hat{e}}{\partial \tau}+ \\
& +\vec{\nabla} H \cdot \frac{\partial \vec{\pi}_{m}}{\partial \tau}+\left(\vec{\nabla} \Phi-\vec{E}^{\prime}\right) \cdot \frac{\partial \vec{\pi}_{\omega}}{\partial \tau}+ \\
& -T \sigma_{s}-\vec{\nabla} T \cdot \vec{j}_{s}-\left(\vec{\nabla} \Phi-\vec{E}^{\prime}\right) \cdot \vec{j}_{\omega} .
\end{aligned}
$$

Here $\hat{e}$ is the Cauchy strain tensor, $\vec{E}^{\prime}=\vec{E}+\vec{v} \times \vec{B}$ and for the increase of kinetic energy in time the expression $d K=\vec{v} \cdot d \vec{k}_{v}$ has been used.

Following the thermodynamics of irreversible processes we can rewrite Eq. (9) in the form:

$$
\begin{aligned}
d U= & T d S+H d \rho+\Phi d \omega+\vec{\nabla} H \cdot d \vec{\pi}_{m}+ \\
& +\left(\vec{\nabla} \Phi-\vec{E}^{\prime}\right) \cdot d \vec{\pi}_{\omega}+\hat{\sigma}: d \hat{e}, \\
\sigma_{s}= & -\frac{1}{T} \vec{\nabla} T \cdot \vec{j}_{s}-\frac{1}{T}\left(\vec{\nabla} \Phi-\vec{E}^{\prime}\right) \cdot \vec{j}_{\omega} .
\end{aligned}
$$

Internal energy of an arbitrarily selected small element of the body is considered to be a thermodynamical potential function of state parameters: entropy $S$, mass density $\rho$ and charge density $\omega$, vectors of elastic displacement of mass $\vec{\pi}_{m}$ and charge $\vec{\pi}_{\omega}$, as well as strain tensor $\hat{e}$, i.e.:

$$
U=U\left(S, \rho, \omega, \vec{\pi}_{m}, \vec{\pi}_{\omega}, \hat{e}\right) .
$$

\section{CONSTITUTIVE EQUATIONS AND THE BASIC FIELD EQUATIONS}

Introducing the Legendre transformation

$$
F=U-T S-\rho H-\omega \Phi-\vec{\pi}_{m} \cdot \vec{\nabla} H-\vec{\pi}_{\omega} \cdot\left(\vec{\nabla} \Phi-\vec{E}^{\prime}\right),
$$

the energy $F=F\left(T, H, \Phi, \vec{\nabla} H, \vec{\nabla} \Phi-\vec{E}^{\prime}, \hat{e}\right)$, for which

$$
\begin{gathered}
d F=-S d T-\rho d H-\omega d \Phi+\hat{\sigma}: d \hat{e}+ \\
-\vec{\pi}_{m} \cdot d(\vec{\nabla} H)-\vec{\pi}_{\omega} \cdot d\left(\vec{\nabla} \Phi-\vec{E}^{\prime}\right),
\end{gathered}
$$

we write such state equations

$$
\begin{gathered}
S=-\frac{\partial F}{\partial T}, \quad \rho=-\frac{\partial F}{\partial H}, \quad \omega=-\frac{\partial F}{\partial \Phi}, \quad \vec{\pi}_{m}=-\frac{\partial F}{\partial(\vec{\nabla} H)}, \\
\vec{\pi}_{\omega}=-\frac{\partial F}{\partial\left(\vec{\nabla} \Phi-\vec{E}^{\prime}\right)}, \quad \hat{\sigma}=\frac{\partial F}{\partial \hat{e}} .
\end{gathered}
$$


Basing on the entropy production expression (11) and Onsager's reciprocal relations, the following kinetic equations can be written

$$
\begin{aligned}
& \vec{j}_{s}=\vec{j}_{s}\left(-\frac{1}{T} \vec{\nabla} T,-\frac{1}{T}\left(\vec{\nabla} \Phi-\vec{E}^{\prime}\right)\right) \\
& \vec{j}_{\omega}=\vec{j}_{\omega}\left(-\frac{1}{T} \vec{\nabla} T,-\frac{1}{T}\left(\vec{\nabla} \Phi-\vec{E}^{\prime}\right)\right),
\end{aligned}
$$

which satisfy the second law of thermodynamics [7].

This yields the definite form of constitutive relations. These relations along with balance equations for total energy, electromagnetic field energy, momentum, mass, entropy and charge, Maxwell's equations for body and vacuum, as well as the Cauchy relation for the strain, make up the complete set of equations in the local gradient model of nonferromagnetic electroconductive solids.

Note that due to classical models which do not regard gradients of chemical and thermodynamical electric potentials as parameters of the local equilibrium state, and supposing the fluxes to be of irreversible form, from (8) we obtain

$$
\frac{\partial \rho}{\partial \tau}=0, \quad \frac{\partial \omega}{\partial \tau}=-\vec{\nabla} \cdot \vec{j}_{\omega}
$$

and that yields to

$$
\rho=\rho_{*}=\text { const }
$$

We assume now function $F$ to be quadratic in disturbances of parameters according to the initial state in the form $H=H_{*}, \rho=\rho_{*}, S=S_{*}, T=T_{*}, \Phi=0, \omega=0, \hat{e}=0$, $\hat{\sigma}=0$. We shall denote disturbances of chemical potential and temperature as $\eta=H-H_{*}$ and $\theta=T-T_{*}$, respectively. This assumption leads to linear state equations. Hence, the linear kinetic relations and the constitutive equations for isotropic body are

$$
\begin{gathered}
\hat{\sigma}=2 \mu \hat{e}+\left[\lambda e-(3 \lambda+2 \mu)\left(\alpha_{t} \theta+\alpha_{m} \eta+\alpha_{\omega} \Phi\right)\right] \hat{I}, \\
s \equiv S-S_{*}=(3 \lambda+2 \mu) \alpha_{t} e-\alpha_{t m} \eta+c_{v} \theta / T_{*}-\alpha_{t \omega} \Phi, \\
\rho-\rho_{*}=(3 \lambda+2 \mu) \alpha_{m} e-\alpha_{t m} \theta-\alpha_{m m} \eta-\alpha_{m \omega} \Phi, \\
\omega=(3 \lambda+2 \mu) \alpha_{\omega} e-\alpha_{t \omega} \theta-\alpha_{m \omega} \eta-\alpha_{\omega \omega} \Phi, \\
\vec{\pi}_{m}=-\chi_{m m} \vec{\nabla} \eta-\chi_{m \omega}\left(\vec{\nabla} \Phi-\vec{E}^{\prime}\right), \\
\vec{\pi}_{\omega}=-\chi_{m \omega} \vec{\nabla} \eta-\chi_{\omega \omega}\left(\vec{\nabla} \Phi-\vec{E}^{\prime}\right) ; \\
\vec{j}_{s}=-\lambda_{s s} \frac{\vec{\nabla} \theta}{T}-\lambda_{s \omega} \frac{\vec{\nabla} \Phi-\vec{E}^{\prime}}{T},
\end{gathered}
$$

$$
\vec{j}_{\omega}=-\lambda_{s \omega} \frac{\vec{\nabla} \theta}{T}-\lambda_{\omega \omega} \frac{\vec{\nabla} \Phi-\vec{E}^{\prime}}{T},
$$

where $\mu, \lambda, \alpha_{t}, \alpha_{m}, \alpha_{\omega}, c_{v}, \alpha_{t m}, \alpha_{t \omega}, \alpha_{m m}, \alpha_{m \omega}, \alpha_{\omega \omega}, \chi_{m m}$, $\chi_{m \omega}, \chi_{\omega \omega}, \lambda_{s s}, \lambda_{s \omega}, \lambda_{\omega \omega}$ are constant coefficients.

Choosing the displacement vector $\vec{u}$, temperate $\theta$, the electric $\Phi$ and chemical $\eta$ potentials as the key functions, we can write the set of field equations in linearized approximation as follows

$$
\begin{gathered}
\mu \nabla^{2} \vec{u}+(\lambda+\mu) \vec{\nabla}(\vec{\nabla} \cdot \vec{u})+ \\
-(3 \lambda+2 \mu)\left(\alpha_{t} \vec{\nabla} \theta+\alpha_{m} \vec{\nabla} \eta+\alpha_{\omega} \vec{\nabla} \Phi\right)=0 \\
\lambda_{s s} \nabla^{2} \theta+\lambda_{s \omega} \nabla^{2} \Phi+ \\
+\frac{\lambda_{s \omega}}{\varepsilon_{0}}\left((3 \lambda+2 \mu) \alpha_{\omega} \vec{\nabla} \cdot \vec{u}-\alpha_{t \omega} \theta-\alpha_{m \omega} \eta-\alpha_{\omega \omega} \Phi\right)=0 \\
+(3 \lambda+2 \mu)\left[\alpha_{m}-\frac{\chi_{m \omega} \alpha_{\omega}}{\varepsilon_{0}}\right](\vec{\nabla} \cdot \vec{u})-\left[\alpha_{m t}-\frac{\chi_{m \omega} \alpha_{t \omega}}{\varepsilon_{0}}\right] \theta+ \\
-\left[\chi_{m m}-\frac{\chi_{m \omega} \alpha_{m \omega}}{\varepsilon_{0}}\right] \eta-\left[\alpha_{m \omega}-\frac{\chi_{m \omega} \alpha_{\omega \omega}}{\varepsilon_{0}}\right] \Phi=0 \\
\lambda_{s \omega} \nabla^{2} \theta+\lambda_{\omega \omega} \nabla^{2} \Phi+ \\
-\frac{\lambda_{\omega \omega}}{\varepsilon_{0}}\left((3 \lambda+2 \mu) \alpha_{\omega} \vec{\nabla} \cdot \vec{u}-\alpha_{t \omega} \theta-\alpha_{m \omega} \eta-\alpha_{\omega \omega} \Phi\right)=0
\end{gathered}
$$

where $\sigma=\hat{\sigma}: \hat{I}$. Such problem allows to describe the nearsurface and nearcontact heterogeneity in nonferromagnetic electroconductive solids after the formulation of the proper boundary and initial conditions.

On the basis of the obtained relations we study some interface phenomena in a layer for isotermal conditions in the sequel.

\section{MECHANICAL AND ELECTRIC FIELDS IN A LAYER}

Let us consider an electroconductive layer (region $|x| \leq l$ in Cartesian coordinates $\{x, y, z\})$. We assume that the values of chemical and thermodynamic electric potentials are given at free of load surfaces $x= \pm l$. The layer can be stretched at infinity $y \rightarrow \pm \infty$. For such conditions in the body one-dimensional situation is realized.

Taking as the key functions the nonzero components of stress tensor $\hat{\sigma}$, the electrical $\Phi$ and chemical $\eta$ potentials and using balance Eq. (17), constitutive Eqs. (18), (19), 
stress equilibrium equation and compatibility equation for one-dimensional situation in the layer, we write

$$
\begin{gathered}
\frac{d^{2} \sigma_{y}}{d x^{2}}=\frac{d^{2} \sigma_{z}}{d x^{2}}=a_{0} \frac{d^{2}}{d x^{2}}\left(\eta+a_{0}^{\omega} \Phi\right), \\
k_{m m} \frac{d^{2} \eta}{d x^{2}}+k_{m \omega} \frac{d^{2} \Phi}{d x^{2}}-a_{m} \eta-a_{\omega} \Phi=0, \\
\frac{d^{2} \Phi}{d x^{2}}+\frac{a_{m \omega}}{\varepsilon_{0}} \eta+\frac{a_{\omega \omega}}{\varepsilon_{0}} \Phi=0,
\end{gathered}
$$

where $a_{0}, a_{0}^{\omega}, k_{m m}, k_{m \omega}, a_{m}, a_{\omega}, a_{m \omega}, a_{\omega \omega}$ are the constants that may be expressed in terms of the constants used in (18), (19) and $a_{m}=a_{0}^{\omega} a_{\omega}$. Here we neglected the stress influence on chemical and electric potentials.

We take now the boundary conditions in the form

$$
\vec{n} \cdot \hat{\sigma}=0, \quad \eta=\eta_{a}, \quad \Phi=\Phi_{a},
$$

at surfaces $x= \pm l\left(\eta_{a} \neq 0, \quad \Phi_{a} \neq 0\right)$, as well as the conditions of mechanical equilibrium

$$
\begin{gathered}
\int_{-l}^{l} \sigma_{y} d x=2 \sigma_{y}^{a} l, \int_{-l}^{l} x \sigma_{y} d x=0, \\
\int_{-l}^{l} \sigma_{z} d x=0, \int_{-l}^{l} x \sigma_{z} d x=0 .
\end{gathered}
$$

We should also take an electroneutrality layer condition

$$
\int_{-l}^{l} \omega(x) d x=0
$$

that leads to constraint $\varphi\left(\Phi_{a}, \eta_{a}\right)=0$ on the surface values of chemical and electric potentials. Another relation that connects these surface values is the balance of mass for the whole body. This produces the conclusion about the selforganizational nature of the body state which means that it is a steady state at the surface of the body.

Neglecting the effect of the electric potential on the chemical potential for the layer which is free of load $\left(\sigma_{y}^{a}=0\right)$, we obtain the problem solution in the form

$$
\begin{gathered}
\eta(x)=\eta_{a} \frac{\cosh \left(\kappa_{m} x\right)}{\cosh \left(\kappa_{m} l\right)}, \\
\sigma_{y}(x)=\sigma_{z}(x)= \\
=a_{0} \eta_{a}\left[\left(1-a_{1}\right)\left(\frac{\cosh \left(\kappa_{m} x\right)}{\cosh \left(\kappa_{m} l\right)}-\frac{\tanh \left(\kappa_{m} l\right)}{\kappa_{m} l}\right)+\right. \\
\left.+a_{2}\left(1+\frac{\kappa_{\omega}}{\kappa_{m}-\kappa_{\omega}}\right)\left(\frac{\cosh \left(\kappa_{\omega} x\right)}{\cosh \left(\kappa_{\omega} l\right)}-\frac{\tanh \left(\kappa_{\omega} l\right)}{\kappa_{\omega} l}\right)\right]
\end{gathered}
$$

$$
\begin{gathered}
\Phi(x)= \\
=\Phi_{a}\left[\left(1+\frac{\kappa_{\omega}}{\kappa_{m}-\kappa_{\omega}}\right) \frac{\cosh \left(\kappa_{\omega} x\right)}{\cosh \left(\kappa_{\omega} l\right)}-\frac{\kappa_{\omega}}{\kappa_{m}-\kappa_{\omega}} \frac{\cosh \left(\kappa_{m} x\right)}{\cosh \left(\kappa_{m} l\right)}\right], \\
\times\left[\left(1+\frac{\kappa_{\omega}}{\kappa_{m}-\kappa_{\omega}}\right) \frac{\cosh \left(\kappa_{\omega} x\right)}{\cosh \left(\kappa_{\omega} l\right)}-\frac{\kappa_{m}^{2}}{\kappa_{\omega}^{2}} \frac{\kappa_{\omega}}{\kappa_{m}-\kappa_{\omega}} \frac{\cosh \left(\kappa_{m} x\right)}{\cosh \left(\kappa_{m} l\right)}\right],
\end{gathered}
$$

where $\kappa_{m}, \kappa_{\omega}, a_{1}, a_{2}, a_{\omega \omega}$ are constants that can be expressed in terms of those used in (21).

This solution demonstrates the existence of two different characteristic sizes of nearsurface heterogeneity in the layer; one is related to the electron subsystem and characterized by parameter $\kappa_{\omega}^{-1}$, while the other is related to the chemical potential (interaction energy) and is characterized by parameter $\kappa_{m}^{-1}$. Comparing the obtained results to those found in literature we can assert that $\kappa_{\omega} \gg \kappa_{m}$.

The results of numerical investigations are demonstrated in Fig. 1, where the distributions of relative electric potential $\Phi^{(r)} \equiv \Phi(x) / \Phi_{a} \quad$ (solid line), charge $\omega^{(r)} \equiv \omega(x) /\left(-a_{\omega \omega} \Phi_{a}\right)$ (dashed line) are presented for $\kappa_{m} / \kappa_{\omega}=0.5, \kappa_{m} l=10$. For large values of $\kappa_{m} l$ (thick layer) the heterogeneity of the charge distribution is inherent in small nearsurface regions, while the inner regions of the body are nearly electroneutral.

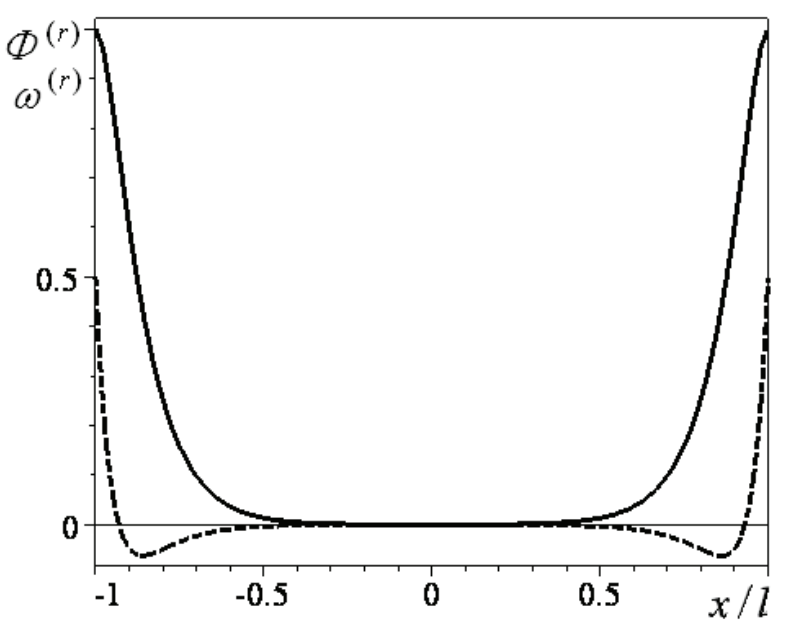

Fig. 1. Electric potential and charge distribution in the layer

\section{SURFACE STRESSES AND ULTIMATE STRESS LIMIT OF A LAYER}

Stress distributions in the layer are similar to the distributions obtained in the framework of the elastic body model for the local gradient approach [8] and the parameter 
$\kappa_{\omega} / \kappa_{m}$ does not essentially influence the stress value. Stress distributions are important for investigating the ultimate stress limit of the body for different conditions of external load. For the stretched layer $\left(\sigma_{y}^{a}>0\right)$ in the considered problem, stresses achieve the maximal value at the surfaces $x= \pm l$ of the body.

Taking the criterion of the first classical theory of strength as a criterion of brittle fracture, i.e. supposing that fracture occurs when the largest principal stress exceeds the given value $\sigma^{f}$, and assuming that the fracture happens instantly when this condition is held in any point of the body from relation $\sigma_{y}( \pm l)=\sigma^{f}$, we obtain the expression for force load intensity $\sigma_{y}^{a}$ that causes the fracture

$$
\sigma_{y}^{a k}=\sigma_{+}+a_{0} \eta_{a}\left((1-k) \frac{\tanh \left(\kappa_{m} l\right)}{\kappa_{m} l}+k \frac{\tanh \left(\kappa_{\omega} l\right)}{\kappa_{\omega} l}\right),
$$

where $k$ is the material constant, and

$$
\sigma_{+}=\sigma^{f}-a_{0} \eta_{a}\left(1-k+k \frac{\kappa_{m}}{\kappa_{\omega}}\right)
$$

is the ultimate stress limit for thick layers $\left(\kappa_{m} l>>1\right.$, $\left.\kappa_{\omega} l>>1\right)$.

Relation (26) shows that with the increase of layer size $l$, the critical load intensity $\sigma_{y}^{a k}$ decreases, which means that the thick layer can bear less net stress than the thin one. Thus the local gradient approach models allow to describe the size effect of the ultimate stress limit. For the size effect two scales are peculiar.

Intensity of the force loading $\sigma^{(r)}$ $\left(\sigma^{(r)} \equiv\left(\sigma_{y}^{a k}-\sigma_{+}\right) /\left(a_{0} \eta_{a}\right)\right)$ that results in brittle fracture dependence on relative thickness of a layer is presented in Fig. 2 for parameters $X=\kappa_{m} l, k=0.5, \kappa_{m} / \kappa_{\omega}=0.2$.

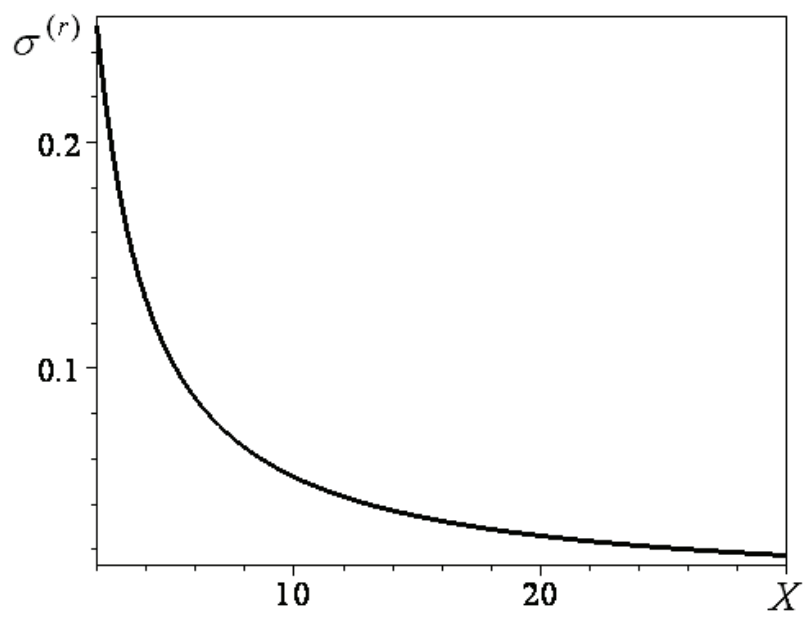

Fig. 2. Critical intensity of force loading dependance on the layer thickness
The surface value of chemical potential (interaction energy) influences the stressed-strained state, ultimate stress limit, and its size effect as well. In [8] the method of finding the surface value of chemical potential in an elastic body is proposed. It is shown that the environment effects, $\eta_{a}$, and consequently the body ultimate stress limit, are essential.

\section{CONCLUSIONS}

In the model of nonferromagnetic electroconductive solid, nearsurface heterogeneity is caused by the electron subsystem of the body, and the variation of interaction energy at the surface results from a given region which comes from all inner regions of the body.

Surface values of chemical and thermodynamical potentials are uniquely determined by physical and geometrical parameters of the body and its environment.

The size effect of ultimate stress limit features two specific sizes (two scales), one of them being related to the electron subsystem of the body, while the other one to the interaction energy.

\section{References}

[1] Ya.Yo. Burak, B.P. Galapats and B.M. Gnidets, Physicalmechanical processes in electroconductive solids. K. Nauk. Dumka. 232 p., 1978 (in Ukrainian).

[2] Ya.S. Podstrigach, Ya.Yo. Burak and V.F. Kondrat, Magnetothermoelasticity of electroconductive solids. K. Nauk. Dumkap. 293, 1982 (in Russian).

[3] Ya.Yo. Burak and T.S. Nahirnyj, Mathematical modelling of local gradient processes in inertial thermomechanical systems. Applied Mechanics 28, 12, 3-23 (1992) (in Russian).

[4] T. Nahirnyj and Y. Burak, Thermodynamical models of continual description of the coupled processes in thin-film systems, Proc. Inter. Symp. on Trends in Continuum Phys. Poznan, Poland. Aug. 17-20, 1998. Word Scientific 263276 (1999).

[5] Ya.Yo. Burak, T.S. Nahirnyj, O.R. Hrytsyna and K.A. Tchervinka, Surface stresses in layer. Effect of temperature and admixture on strength, Problems of strength 6,35-43 (2000) (in Russian).

[6] Ya.Yo. Burak et all., Physical-mathematical modelling of complex systems. Lviv, Spolom. 264 p., 2004 (in Ukrainian).

[7] I. Prigogine, Introduction to thermodynamics of irreversible processes.- Sprinfild, Illinois, U.S.A., 160 p., 1955.

[8] T. Nahirny, O. Hrytsyna and K. Tchervinka, Mathematical modelling and investigation of temperature and admixture effects of strenght, III Sympozjum mechaniki zniszczenia materiałów i konstrukcji. Augustów, Polska, 2005, Bialystok 257-260 (2005). 


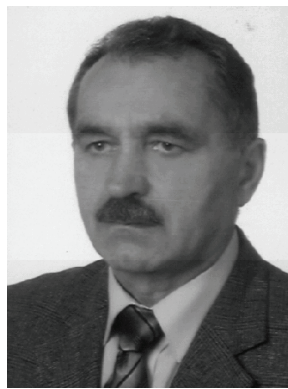

Prof. Taras NahirnyJ was born on June 6, 1951. He received his MSc in Mechanics from the Lviv State University in 1973. He received PhD in mechanics of solids in 1985 from the Institute of Applied Problems of Mechanics and Mathematics named after Ya.S.Pidstryhach of Ukrainian National Academy of Sciences. At present, his affiliation is the Centre of Mathematical Modelling, National Academy of Science of Ukraine, and the Institute of Computer Science and Production Management, University of Zielona Góra. His research interests are mathematical and physical modelling, interrelated processes, computing methods.

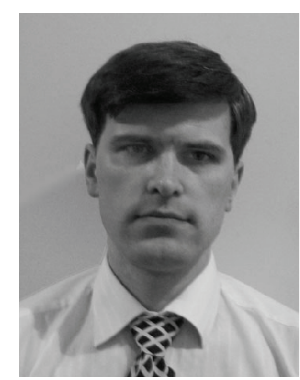

Dr. Kostiantyn TChervinkA was born on June 12, 1976. In 1999 he graduated in Mathematics in Ivan Franko state university of Lviv, Ukraine. He received PhD in mechanics of solids in 2003 from the Institute of Applied Problems of Mechanics and Mathematics named after Ya.S. Pidstryhach of Ukrainian National Academy of Sciences. He works in Ivan Franko national university of Lviv, mechanic and mathematic faculty, mathematical modelling chair. 